\title{
Solution of Active Power Dispatch and Control for Interconnected Power Grids with Large-Scale Wind Power Integration
}

\author{
Zonghe Gao. Xianliang Teng. Xiaobai Zhang. Rui Xu
}

\author{
State Grid Electric Power Research Institute, Nanjing, China (e-mail: gaozonghe@sgepri.sgcc.com.cn).
}

\begin{abstract}
As large-scale wind power has been and will be integrated into power grid, traditional Active Power Dispatch and Control (APDC) system has encountered new challenges. In this paper, the active power dispatch mode of interconnected power grids which is suitable for the large-scale integration of wind power is analyzed firstly. Secondly, it is pointed out that traditional decentralized balance control mode of active power in provincial power grids would not withstand the impact of the large-scale wind power fluctuation. Then, a centralized control mode is proposed in the paper to reallocate all of the generation resources in a wider power grid. Thirdly, the characteristics of the proposed centralized control mode are analyzed from the following aspects: ways of active power balance, mainbodies of active power control, methods of resource allocation, inter-provincial power exchange, security constraints, and data communications. Finally, considering present dispatch system and technical basis in China, a solution is proposed for the active power control for China interconnected power grids. The aim of the proposed solution is to achieve the centralized control mode, and the hierarchical coordination control mode is the transition between present system and the final system.
\end{abstract}

Keywords: Power system control, Wind, Automatic frequency control, Hierarchical control, Integration.

\section{INTRODUCTION}

As an important kind of renewable energy, wind power has developed quite rapidly these years in China. Several colossal wind power bases whose installed capacity is more than millions $\mathrm{KW}$ or even ten millions $\mathrm{KW}$ are under construction in some areas with abundant wind resources. When large-scale wind power is connected to the power grid, its characteristics of randomness and instability will do harm to the safe operation and power quality of power grids, which brings new challenges to the Active Power Dispatch and Control (APDC) system of interconnected power grids (Sun, et al., 2007, Lei,2003).

In order to improve traditional dispatching measures and AGC control modes, we must solve the problems of ADPC system when considering the special characteristics of wind power. On one hand, we must raise the adjustment capability of wind turbines and wind farms to make them participate in the control in proper ways. On the other hand, the large-scale wind power must be digested in the wider areas by utilizing the adjustable resources all through the power grid.

Within the China synchronous grid, the control mode is organised in a hierarchical structure with Provincial Control Area and Regional Control Area, as shown in Fig. 1. Each provincial control area uses Tie-line Bias Control (TBC) mode to maintain the local balance of active power in provincial grids. The regional control areas are usually responsible for frequency regulation in case of emergency or taking on some special control assignments such as regulating the cross-regional UHV AC tie-lines. According to the different dispatch authority, Units can be divided into two types: provincial units and regional directly-controlled units. It should be noted that although regional directly-controlled units are located in provinces, the control authority still belongs to regional control areas (Gao, et al., 2009a).

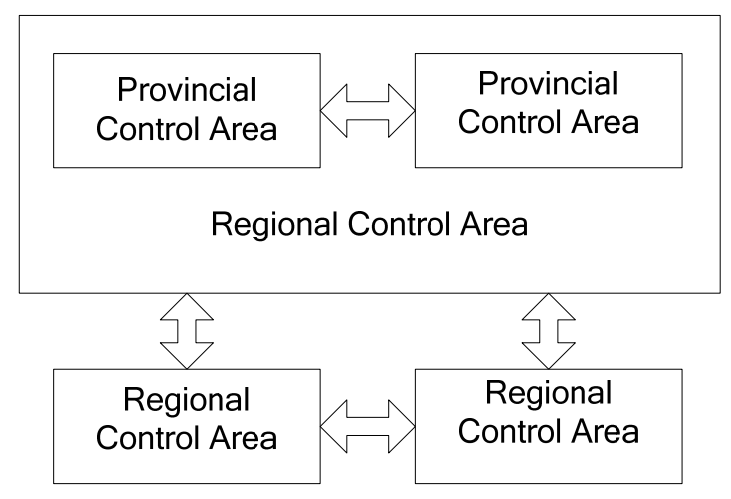

Fig. 1. Hierarchical structure of China synchronous grid.

The control mode of decentralized self-balance in provincial girds requires some specific regulating resources. Any province lacking of this kind of resources often needs to be supported by other adjacent control areas, but the support is limited (Gao, et al., 2005). Moreover, because current control mode of decentralized self-balance is short of coordination, the performance of frequency regulating and power exchange control will not be satisfactory in the case of large disturbances. Meanwhile, due to the limits of dispatch authority, the directly-controlled units of regional grids can not be fully utilized, which means that some regulating resources of high quality are wasted to some extent.

From the above discussion, it can be seen that traditional control mode of decentralized self-balance in provincial grids can no longer satisfy the needs to digest large-scale wind power. Therefore, the study of a new APDC system is of great necessity now. The new mode should be able to fully 
utilize the regulating resources in the regional and provincial power grids, improve the inter-provincial active power support, smooth the fluctuations of wind power and improve the AGC regulating capability.

Firstly, this paper proposes the frame of APDC system for regional and provincial grids with large-scale integration of wind power. Then, the technology of active power control considering wind power is discussed from the aspects of generation scheduling based on wind power prediction and ancillary frequency regulation respectively. Secondly, the centralized control mode that can reallocate all the generation resources in a wider area is proposed. Finally, considering the present dispatching structure and technical basis in China, a solution is proposed for the active power control. The aim of the proposed solution is the centralized control mode, and the hierarchical coordination control mode is the transition between present system and the final system.

\section{APDC SYSTEM CONSIDERING LARGE-SCALE WIND POWER INTEGRATION}

The dispatches of different time scales (including day-ahead [day-inside] dispatch, real-time dispatch and AGC) and the relations among them constitute the framework of traditional APDC system (Teng, et al., 2008). Due to uncertainty of wind power and operation characteristics of wind turbines, power regulating ability of wind turbines can not be compared with that of conventional hydro or thermal units. Therefore, when wind farms are considered in the framework of APDC system, traditional control principle of APDC system should be changed. The new control principle should be centred on the "generation schedule following" mode based on wind power prediction, and be supplemented with "ancillary frequency regulation" mode of wind turbines. The proposed framework of APDC system is shown in Fig. 2.

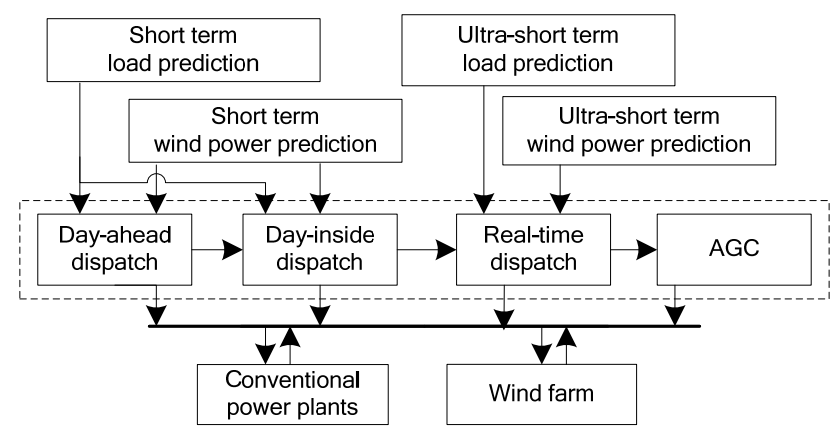

Fig. 2. Framework of APDC system considering large-scale wind power integration.

Compared with traditional APDC system, some new functions should be added when large-scale wind power is integrated.

\section{(1) Short-term and ultra-short-term wind power prediction}

The wind power prediction function can be used for generating short-term or ultra-short-term predictions of the wind power production. It can be configured to cover the total wind power in a region or a single wind farm. The function provides reliable estimates of the uncertainty of the predictions, which is important for optimal scheduling. The short-term wind power prediction is mainly used in day-ahead and day-inside generation scheduling, while the ultra-short term prediction chiefly in real-time generation scheduling.

Optimization for generation scheduling is realized by using wind power prediction in multi-time-dimension in model. At the same time, some factors should be considered in the optimization such as the same time-level load prediction to wind power prediction, network topology, maintenance scheduling, and security constraints. When wind power simply covers a small part of the overall generation capacity, it plays a role in the generation scheduling optimization as the negative load. The prediction deviation is mainly counterbalanced by conventional AGC units. With constant increase of wind power, both wind turbines and conventional units should be considered in the optimization model. Sufficient liquidity in real-time and day-inside dispatching is necessary to absorb large amount of wind power (Weber, 2010). In this circumstance, the generation scheduling is produced through Security Constrained Unit Commitment (SCUC) and Security Constrained Economic Dispatch (SCED).

However, since wind power prediction may cause large deviation, there must be sufficient spinning reserve for conventional AGC units in the optimization model. Moreover considering economy of the power grid operation, more wind power digestion doesn't mean more benefits (Jabr, et al., 2009, Leen, et al., 2010). For one thing, the wind power digestion is at the expense of additional conventional reserve; for another, the digestion capacity is partly influenced by the security constraint of network.

AGC in wind farms control level is responsible for distributing real-time generation scheduling to wind turbines. It makes following control possible through a series of strategies such as output power regulation by adjustment of pitch angles or start-stop procedure of wind turbines. Similar to conventional units, the performance of the wind turbines control can be evaluated quantitatively by regulating rate and response time (Qiao, et al., 2009, Hansena, et al., 2006, Lubosny, et al., 2007).

\section{(2) Ancillary frequency regulation}

Since the output of wind turbines is determined by the wind force, frequency regulation ability of wind turbines is very limited. Then, for frequency regulation, wind turbines can only be used as the assistant of conventional AGC units. Only in an emergency, the limited adjustable ability of wind turbines should be used.

The ways of the involvement of wind turbines in frequency regulation are as follows: (a). Decreasing output in high frequency, which means to abandon some wind energy. (b). Increasing output in low frequency. This means that certain reserve should be always kept, and in normal operating states the wind turbines should be worked below its rated output (Simens, 2008, Singh, et al., 2009). Thus, wind turbines can offer temporary supports when the frequency regulation ability of conventional units is not enough. Here, it should be 
noted that the regulation ability of conventional units should be used preferentially, for the participation of wind turbines in frequency regulation will inevitably sacrifice its economical benefit.

\section{CENTRALIZED CONTROL MODE}

\subsection{Problems of Present Control Mode}

The large-scale inter-provincial wind power transmission has been common in the case of large-scale wind farms integration into power grids. In traditional control mode of decentralized self-balance in provincial grids, areas with higher wind power penetration rate undertake the whole power loss when disturbance occurs. Therefore, the method depending on each provincial AGC adjustment strategy will not only prolong the restoring time of power balance, but also need more reserve of the conventional units.

In addition, the decentralized control in present hierarchical structure is lack of cooperation and coordination between regional and provincial grids. More importantly, excess-adjustment or defective-adjustment of AGC often occurs, which results in great fluctuations of system frequency and tie-line power flows.

As is known, there are some directly-controlled units in provincial control areas, and these units have good regulation abilities. However, under the present dispatching structure, Provincial Dispatch Center (PDC) can not regulate these units freely. Only Regional Dispatch Center (RDC) has the control authority of these units. Then these units can not fully contribute their regulation abilities in the case of large-scale wind power integration.

After the large-scale integration of wind power into grids, above defects will be more obvious since all of the useful resources in grids should be fully used to eliminate the wind power fluctuations. In other words, present hierarchical structure has seriously restricted the adjustment capacity of active power control and wind power digestion. Therefore, it is necessary to make a study on the new active power control mode now.

\subsection{The Technological Essence of the Centralized Control}

With the development of dispatch automation system and highly-concentrated information collection in China, the dispatch centers have possessed the abilities of monitoring, analyzing, early-warning and decision-making in an intelligent way. Compared with PDCs, RDCs do better in information collection and overall planning. Consequently, RDCs should play a greater role in active power control, especially when the decentralized self-balance control mode is gradually transferred to the centralized control mode after the integration of wind power.

China will have completed its construction of 3-C (East-China, North-China and Central-China) UHV synchronized power grid by 2012. The RDC mentioned here may either refer to East-China, North-China or Central-China dispatch center respectively, or even the integrated dispatch center of the 3-C grid. With the completion of this power grid in the future, the interconnection of grids will be closer. The National Dispatch Center (NDC) and 3-C power grid have distinct technological superiority in realizing the integrated dispatch and control of active power.

In the centralized control mode, RDC, as the only dispatch center, takes charge of the power imbalance and distributes the adjustment directly to all the units according to the distribution of the AGC resources. In the process of generation distribution, the adjustment resources of high quality should be utilized first and at the same time various security constraints should be considered, such as power flow equations, generation limit of AGC units, security limit of power flow for flow-gates and transmission lines and so on.

If the unified dispatch of the adjustment resources is realized, the fluctuations of wind power could be eliminated soon. This new mode will break the mechanism of decentralized provincial self-balance, and then the fluctuations of tie-line power exchange will be increased to a certain degree. Thus, previous strict constraints of planned inter-provincial power exchange should be relaxed. Compared with the traditional provincial control mode, the new one has the following characteristics:

\section{(1) Way of active power balance}

Within the traditional hierarchical structure, each provincial grid adopts TBC control. Since the TBC control is just responsible for the provincial grids and its decentralized self-balance, its disturbance restoration highly depends on its own adjustment resources. On the contrary, in the centralized dispatch system, active power balance is applied to the regional grid. Consequently, disturbance in any provincial grid will be restored by the overall adjustment resources of the regional grid, which reinforces the wind power digestion greatly.

\section{(2) Mainbody (Control Area) of active power control}

The centralized control is actually based on an integrated control mainbody. If there are multiple control mainbodies, the competition of different control areas in the regulating process, evaluated by CPC (Control Performance Criteria) or CPS standards, would result in excess-adjustment or defective-adjustment (Gao, et al., 2009b).

Just because of this, some scholars are now devoted themselves to the study of coordinative control between different control mainbodies. However, no matter how well the coordinative control performs, it would not be able to outperform the centralized control mainbody. The centralized control mode can not only eliminate the disorderly adjustment between different control mainbodies, but also consider the network security constraints comprehensively. Therefore, it particularly fits the large power grids with high wind power penetration.

\section{(3) Method of resources allocation}

The centralized control mode realizes the unified allocation of the adjustment resources of the whole grid. There are no distinctions between provincial units and regional directly-controlled units any more in the role of dispatching, 
which is beneficial for all units to work equally in AGC and reduce the cost of the ancillary service as well.

\section{(4) Inter-provincial power exchange}

The centralized control breaks the previous decentralized self-balance of provincial grids, releases the constraints on the tie-line power flows, and strengthens the inter-provincial active power support. In most occasions, proper deviation from the planned power exchange will not threaten the safety of power grids, but when the tie-line power exchange reaches the limit value, the constraints on the limit value (see Point 5 "Security Constraint") must be added in SCD to ensure the safe and steady operation of the power grids.

It may enlarge the margin of deviation of the power exchange if the constraints on the tie-line flows are relaxed. It requires monitoring the actual power exchange within a certain period (such as one day or one month). If the deviation is large, the regulating priority of provincial grids depending on the power exchange should be considered in the control allocation.

\section{(5) Security constraint}

The centralized control is beneficial to the elimination of the power imbalance caused by the fluctuations of large-scale wind power, but at the same time it will lead to great changes of network flow and heavy-load or even over-load of the sections. The preventive and remedial control of cross sections flow can be developed by integrated AGC with Security Constrained Dispatch (SCD) to constitute a close-loop control system. Generally, SCD starts in a cyclical manner (such as 1-5 minutes), and gives the generation limit of the AGC units to avoid the violations of the constraints. When there are violations, it starts in an event-driven manner, and provides the remedial control strategies for AGC. With reference to the results of SCD, AGC adjusts the generation to ensure the security and stability of power grid (Zhang, et al., 2005).

\section{(6) Data communication}

In the previous hierarchical structure, each control area has its own AGC independently, so the communication among the PDCs is not very important. However, in the proposed centralized control mode, reliable data communication is absolutely important. The dispatch center needs to collect much more data such as the tie-line power exchange, the power flow of cross sections and the regulating information of AGC units. Data exchange turns out to be more complicated than before.

\subsection{Ways to Realize the Centralized Control}

As shown in Fig. 3, the regional area is responsible for the overall allocation of AGC resources, and positions of the units of provincial grids and regional grid are just the same. Thus, it can be considered that there is only one control area. The control commands can either be distributed directly by RDC to all units, or re-transmitted through PDCs.

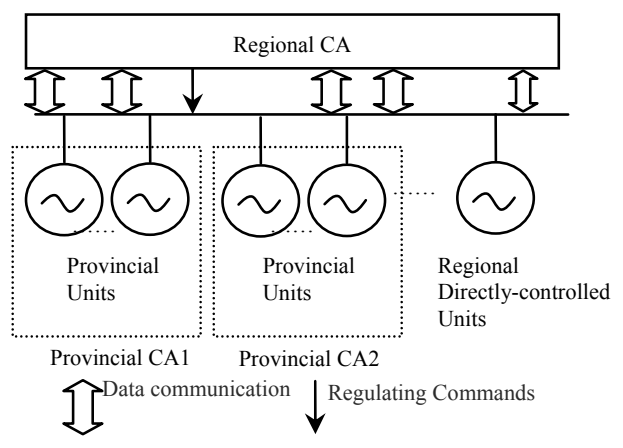

Fig. 3. Centralized control mode for regional grid.

In the centralized control mode, PDCs are still very important. On one hand, PDCs receive the control commands from RDC, and then distribute them to corresponding AGC units after check. On the other hand, PDCs should qualify themselves with completed AGC functions all the same, the reasons of which are as follows: (1) To act as back-up means of control when malfunctions (such as EMS breakdowns of the master stations, communication failures, etc.) occur in the centralized control mode. (2) To control some particular units, such as the units following the scheduled curve.(3) To be applied to some special conditions, such as some specific cross sections.

\section{THE HIERARCHICAL COORDINATION CONTROL MODE}

The hierarchical coordination control mode still keeps PDC and RDC as two independent control mainbodies. On the basis of whether to maintain present decentralized provincial self-balance mode or not, the hierarchical coordination control mode can be divided into two modes: the new provincial decentralized self-balance mode and the unified balance mode.

\subsection{The New Provincial Decentralized Self-balance}

Except in some emergencies, the new provincial decentralized self-balance mode still follows the planning of tie-line power exchange among provinces. The regional directly-controlled units will be regarded as a part of the adjustment resources of PDCs and work together with the provincial units to overcome the active power imbalance in each province. RDCs are responsible for the cooperation and coordination of the regional directly-controlled units and the provincial units. The following are steps of implementation:

(1) RDC calculates the ACE of each provincial control area (as shown in the Dashed frame of Fig. 4) in every AGC cycle.

(2) PDC keeps the outlet lines of the regional directly-controlled units as the general tie-line, establishes the enclosed control model, computes periodically the adjusting ability of the provincial AGC units (including the overall adjusting rate and range) and reports it to RDC in real time.

(3) RDC, considering the adjusting ability of regional directly-controlled units and the reported data in step (2), disintegrates the ACE in step (1) into two parts: one part is distributed to directly-controlled units, the other to PDCs. 
(4) After receiving the ACE, each PDC distributes it to its AGC units according to certain principles.

The new provincial decentralized self-balance control mode is shown in Fig. 4.

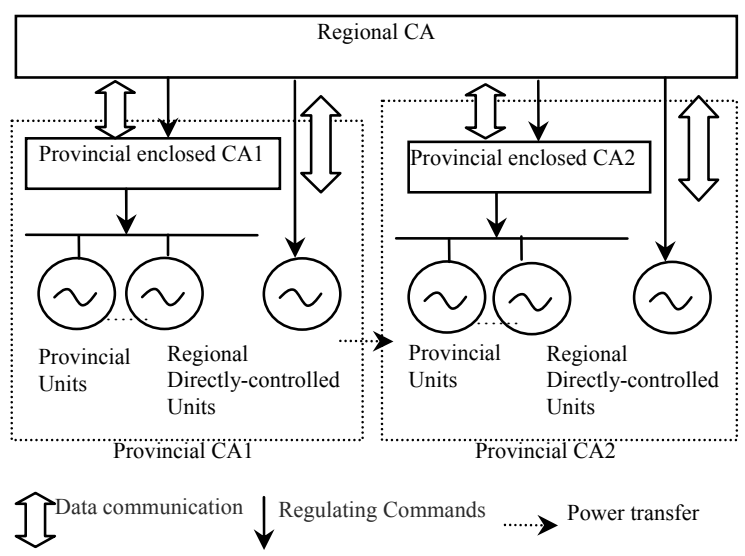

Fig. 4. Hierarchical coordination control mode 1 (new provincial decentralized self-balance mode).

When disintegrating the ACE in step (1), the provincial units should have the priority in adjustment, while the regional directly-controlled units only give support when necessary.

If necessary, a provincial control area is allowed to remove part of its power imbalance to another provincial control area by following certain rules. Because RDC controls all the adjustment resources, the ways to remove the power imbalance can be varied. The choice is determined by specific requirements of different power grids. It should be noted that when removing the power imbalance, it may bring about drastic fluctuations of inter-provincial power exchange, so it is very important to prevent the power exchange from exceeding the security limit value.

\subsection{Unified Balance Mode}

In the unified balance mode, it is no longer necessary to strictly follow the inter-provincial planned power exchange. Only the demand of power exchange for a longer period of time (such as one day or one month) should be satisfied.

In the unified balance mode, the provincial AGC units show themselves as a model of equivalent unit in RDC, as is shown in Fig.5. Both the regional and provincial units share the active power imbalance. Similar to the new provincial decentralized self-balance mode, PDC needs to upload overall information of AGC units to RDC in real time, such as regulation speed and range of equivalent unit. $\mathrm{RDC}$ should calculate the adjustments of directly-controlled units and equivalent units in real time and download the adjustment of equivalent units to corresponding PDC. Then, ACE from RDC would be distributed to each AGC units by the provincial AGC.

The unified balance control mode is shown in Fig. 5.

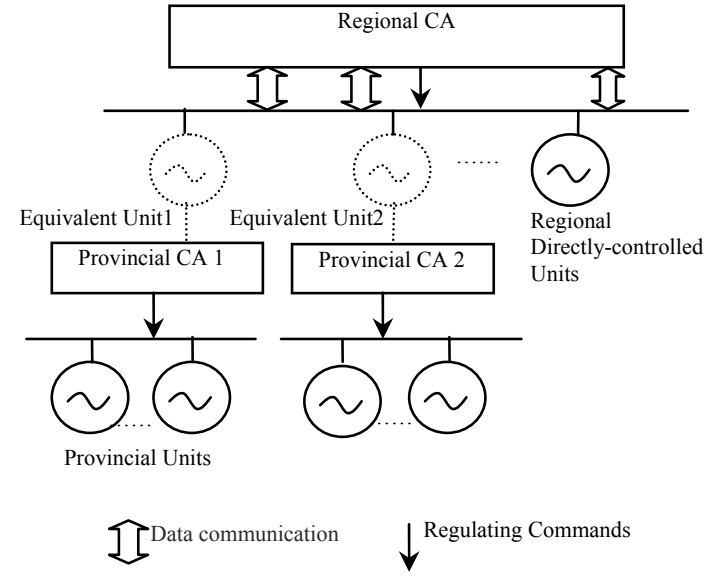

Fig. 5. Hierarchical coordination control mode 2 (unified balance mode).

When RDC distributes the regulating allocation of the units, the security constraints of the tie-lines and the relevant cross sections should be fully considered.

\section{CONCLUSIONS}

The centralized control mode is an ideal control mode for power grid integrated with large-scale wind power, for this mode can efficiently consider the security constraints of whole power grid and can realize the optimal dispatch of AGC resources in a wider range. However, presently there still exist two difficulties in the implementation.

(1) The application of this mode highly depends on the support of high-level informational and automatic technologies (Yao, et al., 2010). However, presently the construction of a coordinated control system focused on information concentration, intelligent decision-making and integrated control is very complex and time-consuming.

(2) The transformation from traditional decentralized self-balance mode to centralized dispatch mode means that present hierarchical structure of active power control should be broken. Then, new dispatch rules should be developed, and this can not be accomplished in a short time.

Since the above two aspects need more time to realize, a three-step strategy as follows is proposed. To achieve the smooth transition both in technology and management, the aim of the proposed strategy is the centralized control mode, and the hierarchical coordination control mode is the transition between present system and the final system.

Step I, the application of hierarchical mode 1 (the new provincial decentralized self-balance control mode), which is simple for the implementation and can achieve the efficient coordination between regional and provincial AGCs.

Step II, the application of hierarchical mode 2 (unified control mode), which aims to strengthen the coordinative function of regional grids and reduce the waste of resources in the decentralized control system. 
Step III, the application of the centralized control mode, which helps to remove the technological barriers in the integration and the digestion of the large-scale wind power.

Acknowledgement: This work was supported by the National Mega-Projects of Science Research for the 11th Five-year Plan of China (No.2008BAA13B06).

\section{REFERENCES}

Gao, Z.H., Teng, X.L., and Zhang, X.B. (2005). Automatic generation control strategy under control performance standard for interconnected power grids. Automation of Electric Power Systems, 29(19), 40-44.

Gao, Z.H., Chen, G., and Yang, J.F. (2009a). Active power control for tie-lines in UHV interconnected power grid part one AGC control strategies. Automation of Electric Power Systems, 15, 51-55.

Gao, Z.H., Chen, G., and Teng, X.L. (2009b). Active power control for tie-lines in UHV interconnected power grid part two AGC performance assessment. Automation of Electric Power Systems, 16, 61-64.

Hansena, A.D., Sørensena, P., Iovb, F., and Blaabjerg, F. (2006). Centralized power control of wind farm with doubly fed induction generators. Renewable Energy, 31,931-951.

Jabr, R.A., and Pal, B.C. (2009). Intermittent wind generation in optimal power flow dispatching. IET Generation, Transmission \& Distribution, 3, 66-74.

Leen, V., Leonardo, M., Ronnie, B., Marcelo, S., and Jean, M.G. (2010). Well-functioning balancing markets: A prerequisite for wind power integration. Energy Policy, $38,3146-3154$

Lei, Y.Z. (2003). Studies on wind farm integration into power system. Automation of Electric Power Systems, 27(8), 84-89.

Lubosny, Z., and Bialek, J.W. (2007). Supervisory Control of a Wind Farm. IEEE Transactions on Power Systems, 22, 985-994.

Qiao, Y., and Lu, Z.X. (2009). Wind farms active power control considering constraints of power grids. Automation of Electric Power Systems, 22, 88-93.

Siemes, P., Haubrich H. J., Vennegeerts, H., and Ohrem, S. (2008). Concepts for the improved integration of wind power into the German interconnected system. IET Renewable Power Generation, 2, 26-33.

Singh, B., and Singh, S.N. (2009). Wind Power Interconnection into the Power System: A Review of Grid Code Requirements. The Electricity Journal, 22, 54-63.

Sun, Y.Z., Wu, J., and Li, G.J. (2007). Influence research of wind power generation on power systems. Power System Technology, 31(20), 55-62.

Teng, X.L., Gao, Z.H., and Zhang, X.B. (2008). Look-ahead control for active power dispatching system and online hydro-thermal coordinated control strategy. Automation of Electric Power Systems, 32(22), 16-20.

Weber, C. (2010). Adequate intraday market design to enable the integration of wind energy into the European power systems. Energy Policy, 38, 2334-2343.
Yao, J.G., and Lai, Y.N. (2010). The essential cause and technical requirements of the smart grid. Automation of Electric Power Systems, 2, 1-4.

Zhang, X.B., Gao, Z.H., and Qian, Y.M. (2005). Implementation of preventive and remedial control for tie line overload by use of automatic generation control. Power System Technology, 29(19), 55-59. 